\title{
From mink to malaria
}

\author{
Francine Ntoumi is a Congolese parasitologist, founder, chair and executive director of the Congolese Foundation \\ for Medical Research, Republic of Congo, and research group leader at the University of Tübingen, Germany. She \\ established the first research centre on infectious diseases in the Republic of Congo.
}

\section{Francine Ntoumi}

rom a young age, I have enjoyed puzzles and detective stories - the questioning, putting pieces together to solve a problem, and trying to guess the culprit based on the available clues. I have also always been interested in microbiology. It therefore made perfect sense to pursue a scientific career. Today, I am a molecular epidemiologist working on infectious diseases in the Republic of Congo, studying the identification of a criminal - a microbial pathogen - that uses a very subtle strategy to defeat human immunity, and even to kill. My role as a principal investigator includes laboratory research, profiling the type of pathogen to be investigated, teaching and negotiating funding.

In 1995, after receiving a $\mathrm{PhD}$ in physiology from the University Paris VI in France, following four years investigating the circadian rhythm of minks (Mustela), I asked myself two questions. First, what disease has the highest mortality rate and represents one of the biggest challenges for public health in Africa? My goal was to return home to Congo. Second, where would I be able to acquire the necessary knowledge that would enable me to tackle this disease and be of use to my country? The disease in question is malaria, and for me, the Pasteur Institute in Paris was the place where I wanted to receive my training. This was my first turning point. In this famous research centre, I immediately noticed the fusion of passion, knowledge and doubt that scientists apply to solve problems. The clash of ideas, the presentation of many hypotheses and the art of argumentation was my daily training. I learnt that a solution to a scientific problem can take a long time and that our work usually represents just a small, yet important, step forward in our efforts to tackle diseases such as malaria. I also discovered the humility of many researchers. They were aware of the incompleteness of their knowledge, as someone else could question their theory. Therefore, everything was stated very

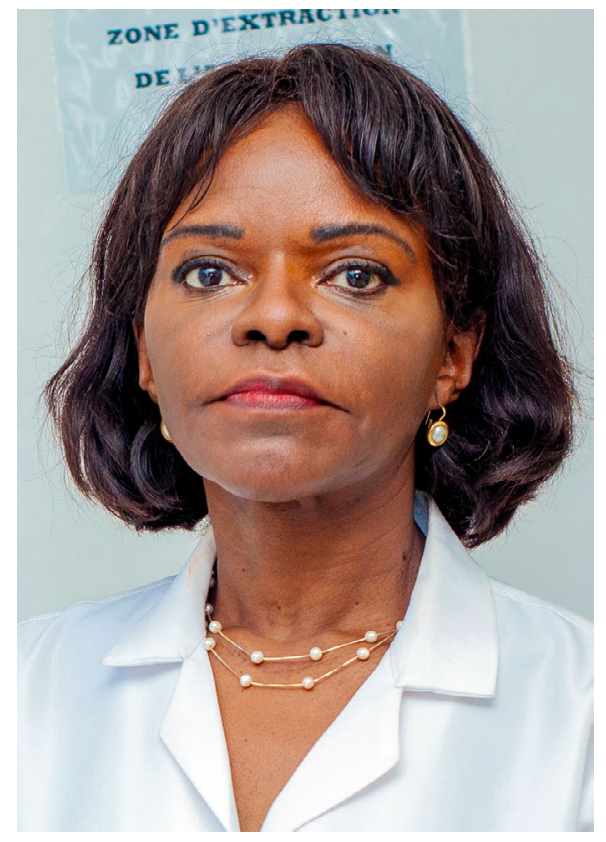

Credit: Fondation Congolaise pour la Recherche Médicale

carefully, including when publishing in the best scientific journals.

I took this knowledge from the Pasteur Institute to Gabon, where I got my first position as a researcher. I focused my work on studying the genetic diversity of Plasmodium falciparum isolates collected from different geographical contexts (Senegal, Cameroon, Gabon and the Republic of Congo) and various clinical samples. Human genetic background and immune response were considered as important variables. I have since continued this work as a principal investigator, with my team showing that the genetic diversity of malaria parasites infecting carriers of the sickle cell trait (N. S. Gampio Gueye et al. Malar. J. 18, 57; 2019 and F. Ntoumi et al. Exp. Parasitol. 87, 39-46; 1997) - which is common in sub-Saharan Africa - was different to that of parasites infecting hosts with normal haemoglobin, and that this could contribute to the mechanism of protection against clinical malaria. Back to Congo, as there was no molecular biology laboratory at the only public university, I created one.

Recently, for the first time, a vaccine against malaria will be deployed in sub-Saharan Africa. This is a significant victory, but there are challenges due to its low efficacy, highlighting the need for continued research in this area. Meanwhile, the COVID-19 pandemic continues to cause record numbers of cases and deaths. According to the World Malaria Report, a total of 241 million cases and 627,000 deaths due to malaria have been reported at the time of writing, with $82 \%$ of cases and $95 \%$ of deaths reported from Africa. The availability of financial resources where malaria is endemic, including sub-Saharan Africa, is a key limitation for research against this disease. An annual investment gap of US $\$ 3$ billion is faced and the contribution of malaria-endemic countries has remained unchanged for several years.

No matter how hard scientists work, in most cases, the impact of their findings will almost always be limited to their immediate academic circles, and the findings do not usually catch the attention of those who have the power to act on them. This is most acute in malaria-endemic countries, where the disease is slowly decimating the well-being of the population and the national economies. This is also reflected by the lack of specialized laboratories and qualified personnel, and the very limited national financial support for health research. It is not surprising that disciplines such as microbiology, entomology and epidemiology are not attractive to the younger generation. This realization was my second turning point.

There are two dates in my journey that have allowed me to catch the attention of those with the power to make a difference to people's lives.

On 4 March 2011, an explosion occurred in an arms depot in Brazzaville, Congo, with more than 300 deaths - many of which were unidentifiable. I felt that everyone who could, should try to help. Being a molecular 
biologist, I went from studying the DNA of the malaria parasite to human DNA fingerprinting in cooperation with the Red Cross legal practitioner to help identify the victims of the explosion. This has allowed many Congolese families to mourn and come to peace with losing their loved ones. This had a great emotional impact on me and was an unforgettable experience allowing me to help others. It also allowed me to show my fellow citizens the value of research and the need for qualified resources and a well-equipped laboratory locally.

Since the beginning of 2020, my laboratory has helped tackle the SARS-CoV-2 pandemic, which is spreading in Congo-Brazzaville as it is in the rest of the world. It is the only laboratory in the country capable of viral sequencing and has thus been invaluable to decision-makers (F. Ntoumi Nature 587, 331; 2020 and F. Ntoumi et al. Int. J. Infect. Dis. 105, 735-738; 2021). The first sequence of the B.1.640 variant from the Republic of Congo was published by my team at the end of August 2021 on the GISAID platform and led to its classification as a variant under monitoring by the World Health Organization. This shows that Congolese research is of global importance and should therefore be valued and supported by those in power.

Years of passionate research have passed, yet the battle against infectious diseases - including malaria - continues to be fought. My first turning point was to study malaria. I hope that the next turning point in my career will be to contribute to having research in Africa recognized as a priority by local and global stakeholders alike.

Francine Ntoumi (1D) $1,2 \bowtie$

${ }^{1}$ Fondation Congolaise pour la Recherche Médicale (FCRM), Brazzaville, Republic of Congo. ${ }^{2}$ Institute of Tropical Medicine, Universitätsklinikum Tübingen, Tübingen, Germany.

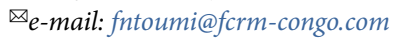

Published online: 4 March 2022

https://doi.org/10.1038/s41564-022-01062-7

Competing interests

The author declares no competing interests. 